\title{
Modeling of Crystallographic Texture Formation in Hot-rolled Sheets of Ferritic Steel
}

\section{Yu. Perlovich, M. Isaenkova, P. Dobrokhotov, D. Zhuk, and A. Rubanov}

National Research Nuclear University MEPhI (Moscow Engineering Physics Institute), Kashirskoe shosse 31, Moscow, 115409, Russia

\section{ANNOTATION}

It is known that hot rolling of ferritic steel leads to formation of texture inhomogeneous through sheet thickness [1-3]. For analysis of processes, which induce formation of texture inhomogeneity, finite element method simulation of hot rolling was carried out at macro- and mesoscopic levels. Crystallographic texture is a sensitive indicator of plastic deformation process so it is possible to use it to assess the quality of the simulation. Simulated orientation distribution functions are in a good agreement with experimental result in central part of sheet (middle in thickness), but does not substantially correspond with it in outer layer. Reasons of these inconsistencies were

Corresponding Author:

A. Rubanov

toly.rubanov@yandex.ru

Received: 21 December 2017

Accepted: 15 April 2018

Published: 6 May 2018

Publishing services provided by Knowledge E

(c) Yu. Perlovich et al. This article is distributed under the terms of the Creative Commons

Attribution License, which permits unrestricted use and redistribution provided that the original author and source are credited.

Selection and Peer-review under the responsibility of the MIE-2017 Conference Committee. analyzed and following were singled out: disconnection of texture with mechanical properties, possible operation of some other mechanism of deformation apart from crystallographic slip.

\section{INTRODUCTION}

Inhomogeneous texture is observed in hot-rolled sheets of $\mathrm{Fe}-0,55 \% \mathrm{C}-1 \% \mathrm{Cr}-2 \% \mathrm{Ni}$ $1 \%$ Mo steel: outer layer and central part characterized by components $\{112\}<111>$, $\{110\}<112>$ and $\{100\}<110\rangle,\{112\}<110\rangle,\{554\}<225>$ respectively [1]. It is assumed that this inhomogeneity is due to singularities of temperature and deformation fields induced by more intensive cooling of outer layer and friction in sheetroll contact. There are two hypotheses about surface texture formation. In [2] it is supposed that texture formation in outer layer occurs under the action of shear deformation near the surface of the sheet. However, in [3] developed the concept of anomaly texture formation with a high role of dislocation climb under conditions of surface hardening by interstitial impurities, which diffused from the surface of the sheet at high temperature and contact with air. Therefore, the aim of the work is to simulate the process of steel hot rolling to identify factors that introduce the inhomogeneity of the crystallographic texture in hot-rolled sheets.

\section{S OPEN ACCESS}




\section{MATERIALS AND METHODS}

Finite element method was used for simulation of hot rolling process on macro- and mesoscopic levels. Rolling modeling was carried out separately for each rolling step, which characterized by thickness of sheet after passing through rolls (table 1). The scheme of the relationship between different levels of the hot rolling process modeling is shown in Figure 1.

TABLE 1: Change in sheet thickness during rolling.
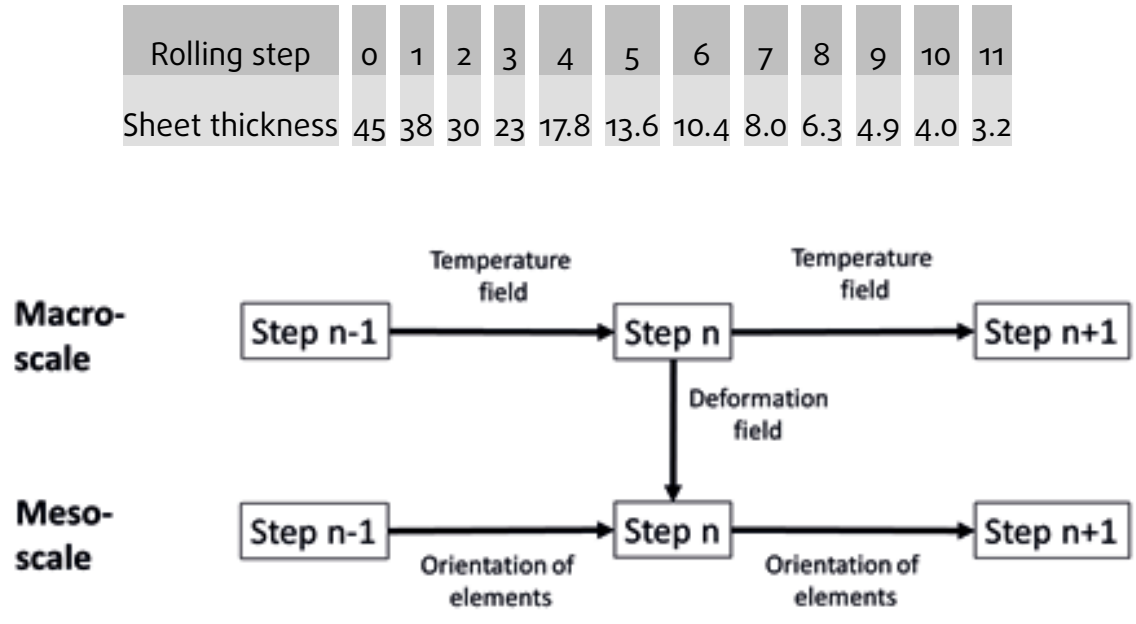

Figure 1: Scheme of the hot rolling process modelling.

At the macroscopic level, the simulation was carried out by direct couple termostructure analysis in a two-dimensional formulation considering the middle plane of the sheet symmetry. An example of the model geometry for the first rolling pass with the mesh is shown on Figure 2.

The material was considered homogeneous and isotropic. The dependences of the yield strength, heat capacity, and thermal conductivity on temperature were set. The model takes into account the oxide layer effect on the metal surface heat exchange by setting the appropriate properties (heat capacity, thermal conductivity) for the nodes on the outer surface of the sheet. As boundary conditions for the heat equation, heat exchange with the surrounding environment was determined by convection and radiation and thermal conductivity in contact with the rolls. An additional heating of the rolled sheet due to plastic deformation was taken into account. The initial temperatures of the rolls and sheet are set before the start of rolling as $200^{\circ} \mathrm{C}$ and $1100^{\circ} \mathrm{C}$ respectively. In subsequent rolling passes, the temperature field from the previous stage of deformation was used as the initial temperature distribution.

For mechanical analysis the roll was rotated about its axis. Roll interaction with the rolled sheet was contact with friction. At the transition from one pass to the next, strain 


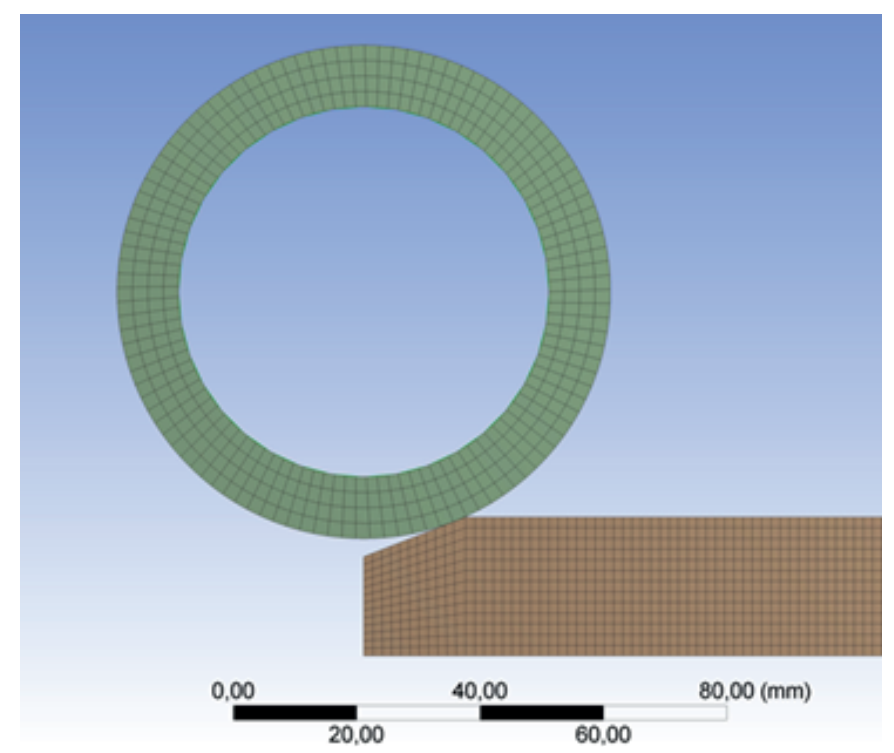

Figure 2: Geometry model with the mesh for first rolling pass.

hardening was not taken into account because of the annealing of the defects at high temperature (i.e., there is no hardening of the material at the beginning of each rolling step).

On mesoscopic level the calculation of grain reorientation was carried out for each rolling step by cube of $8 \times 8 \times 8$ material points. For boundary conditions deformation field from macroscopic level simulations was used. Plastic properties of material were given by phenomenological dislocation-based model [4] with slip systems $\{110\}<111>$ and $\{112\}<111>$ for ferrite and $\{111\}<110>$ for austenite. Relaxed grain clusters (RGC) [5] homogenization scheme with eight elements in cluster was applied. On the first rolling step random initial orientation were chosen and at each subsequent rolling step the orientation of the elements was set from the previous one. Figure 3 shows the distribution of the Euler angle $\varphi_{1}$ after the eighth pass.

For texture changes analysis orientation distribution function (ODF) was calculated. In our case it is convenient to represent the ODF by cross section at $\varphi_{2}=45^{\circ}$, which contains the main texture components characteristic for materials with a cubic crystal structure. The schematic arrangement of typical texture components is shown on Figure 4.

\section{RESULTS}

As a result of macroscopic simulation the distributions of the strain tensor components for different sheet layers and the distribution of temperatures at each rolling step are obtained. The parameter $S$, which is given by the relative distance to the sheet center 


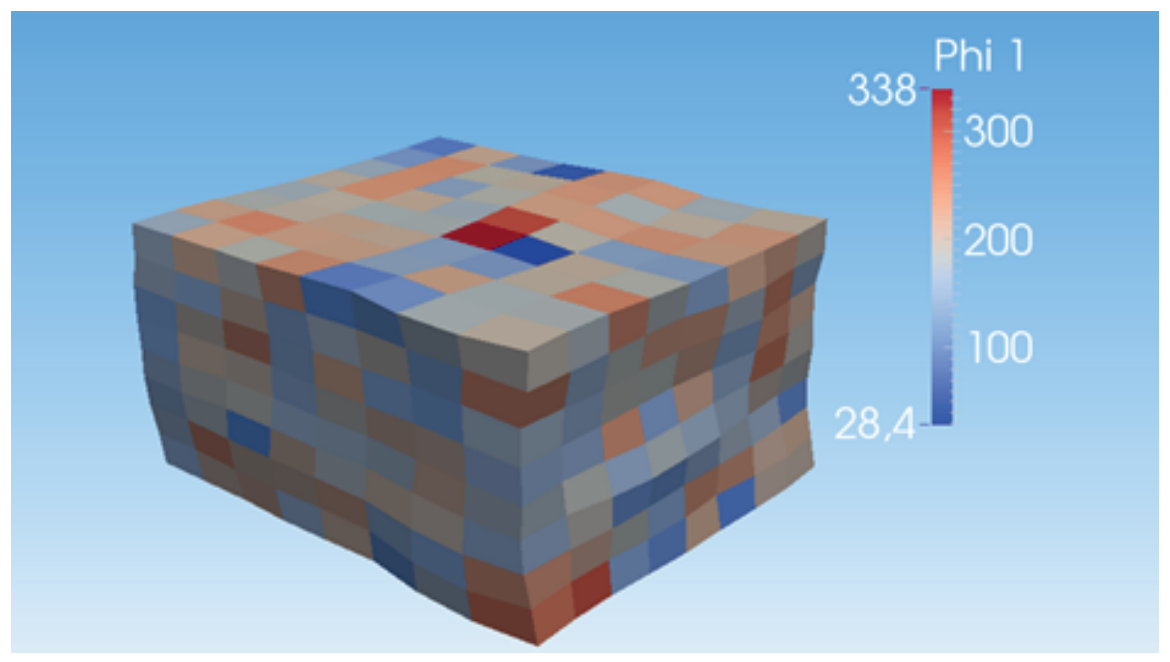

Figure 3: Euler angle $\varphi_{1}$ distribution after the $8^{\text {th }}$ rolling step.

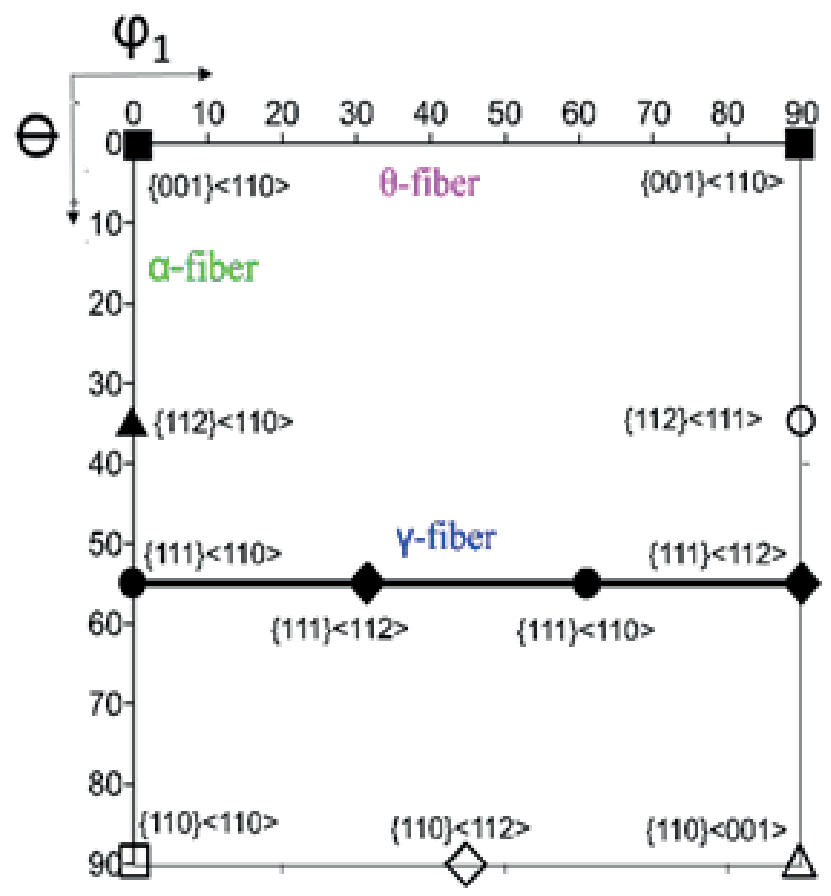

Figure 4: The arrangement of typical texture components on ODF cross section at $\varphi_{2}=45^{\circ}$.

along its thickness $S=\left(t_{0}-t\right) / t_{0}$, where $t$ is the distance from the surface of the sheet and $2 t_{0}$ is the initial thickness of the sheet, is used as the coordinate specifying the position in the sheet section. Figure 5 (a) shows the distribution of temperature from the number of the pass and the rolling time, while fig. 5 (b, c, d) shows the distribution of strain tensor components for different values of parameter $S$. It can be seen that the near-surface layers of the sheet cools down faster than the central layers, and the majority of the time it is in the ferrite temperature range (below the straight line $\left.A_{1}\right)$. The component $\varepsilon_{x}$ of the strain tensor varies little over the entire thickness of the sheet, while $\varepsilon_{y}$ and $\varepsilon_{x y}$ in different layers have different values. It should be noted that 
at the $2-4$ steps of rolling the shear component of the strain tensor in the near-surface part of the sheet is greater than the normal components.

a

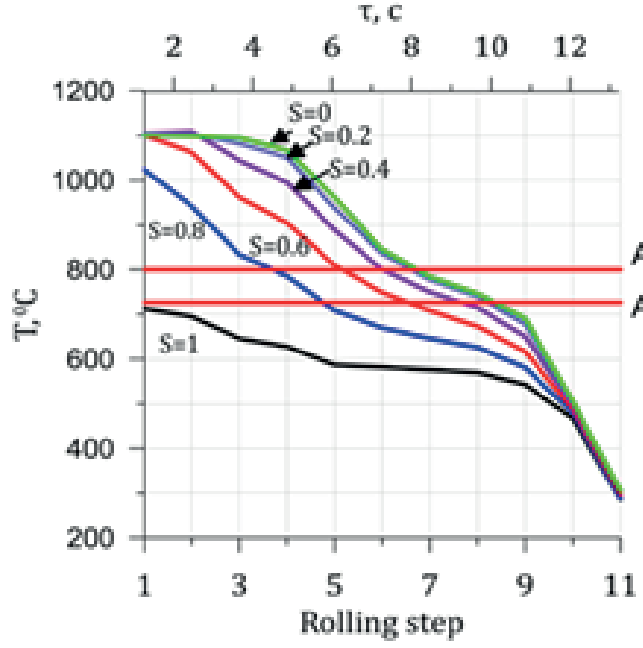

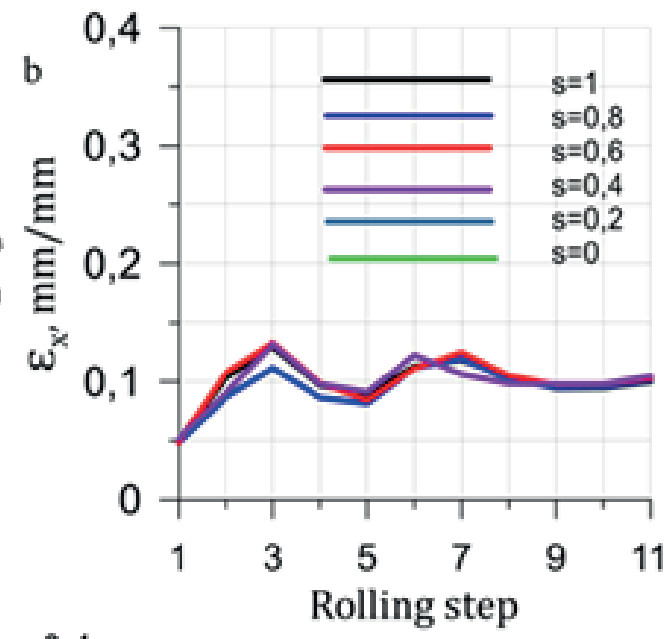

c
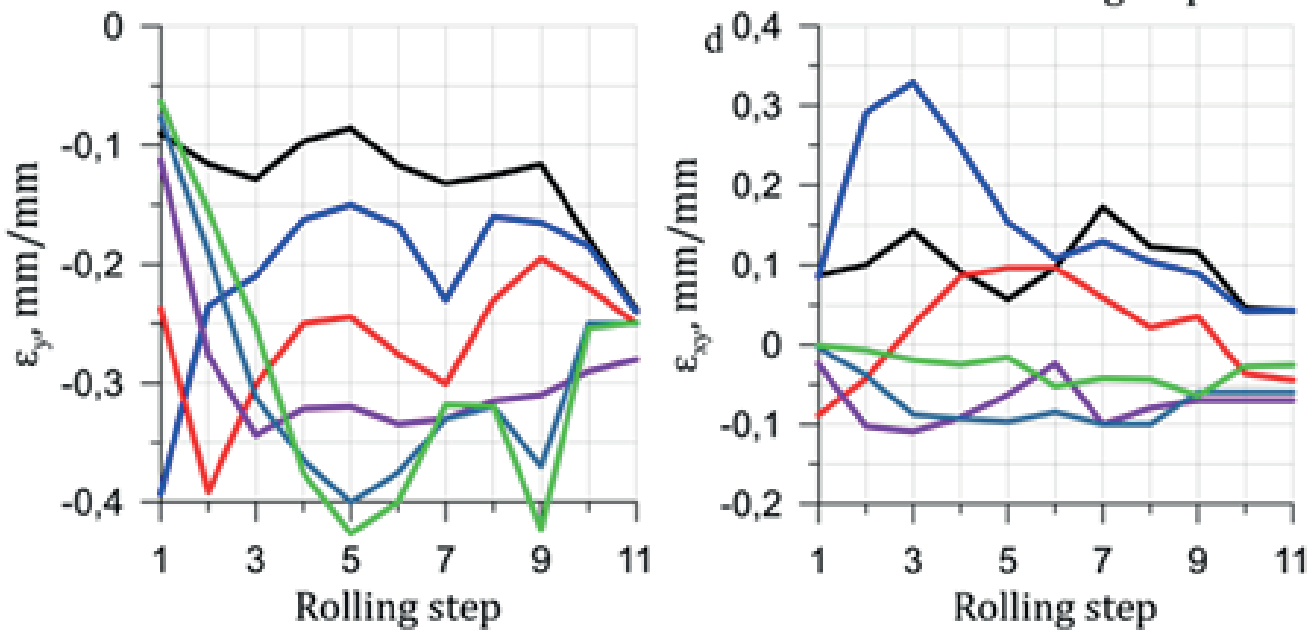

Figure 5: Distribution of temperature (a) and strain tensor components (b, c, d) for successive stages of rolling and various parameters $S$.

With deformation tensor components (fig. 5) mesoscopic simulation was carried out. Strain tensor for central $(S=0)$ and outer layer $(S=0.9)$ was chosen for calculation.

In central layer, first eight rolling steps take place in austenitic temperature range, and other steps take place in ferritic. Thus, the first eight passages were modeled using the $\{111\}<110>$ slip systems for austenite, after which the orientations of the elements were recalculated in accordance with the Kurdjumov-Sachs orientation relations $\{111\} \gamma / /\{011\} \alpha$ and $<011>V / /<111>\alpha[6]$. On the remaining stages the slip systems $\{110\}<111>$ and $\{112\}<111>$ for ferrite were used. Based on the simulation results, the ODF cross sections at $\varphi_{2}=45^{\circ}$ for the austenitic temperature range are shown in Fig. 6 . 


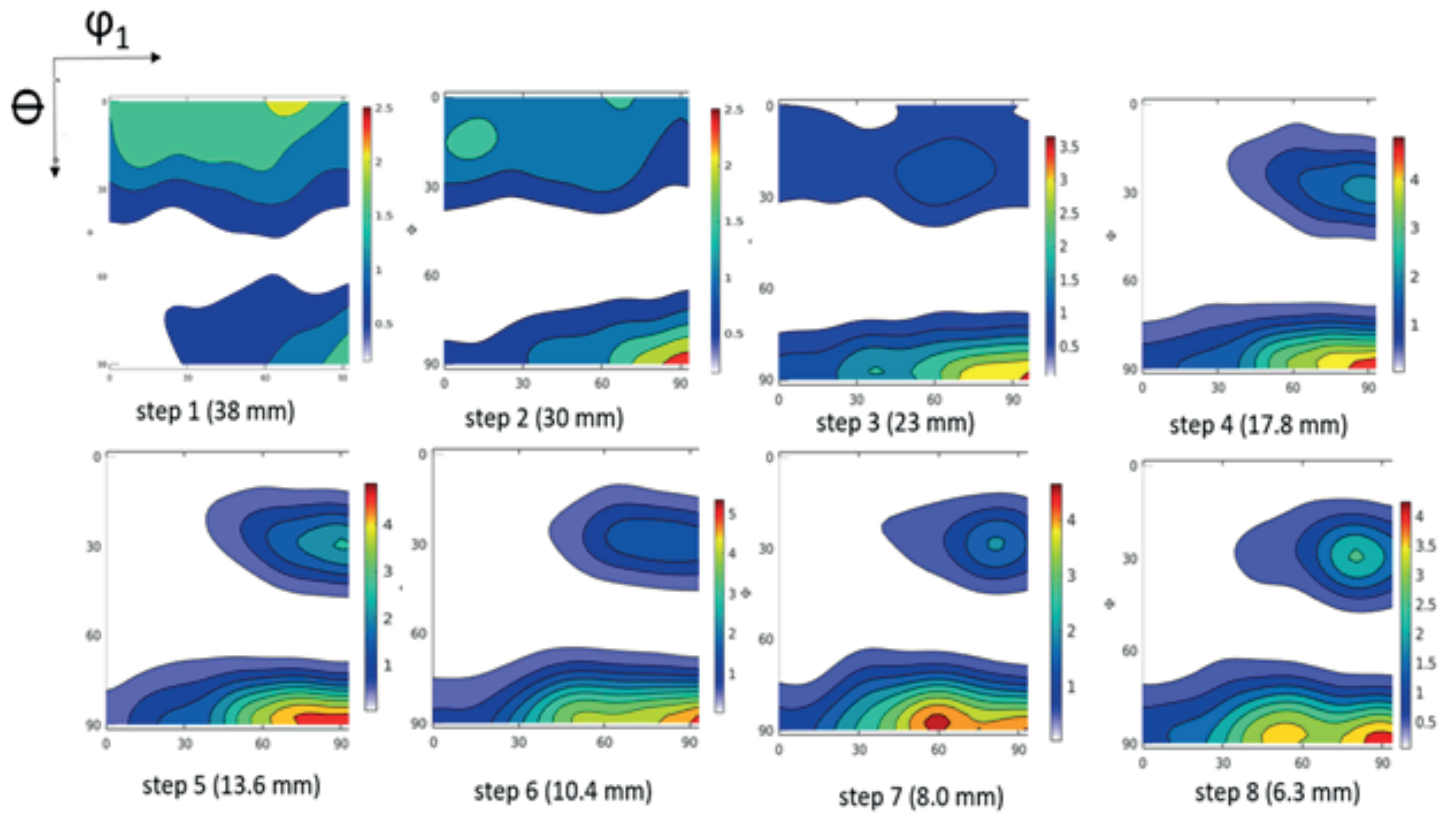

Figure 6: Simulated ODF sections $\varphi_{2}=45^{\circ}$ for rolling steps in austenitic range of temperature (central layer).

Figure 7 shows simulated ODF sections for ferritic temperature range. Experimental texture components for austenite (a) and ferrite (b) after hot rolling are placed on Figure 8.
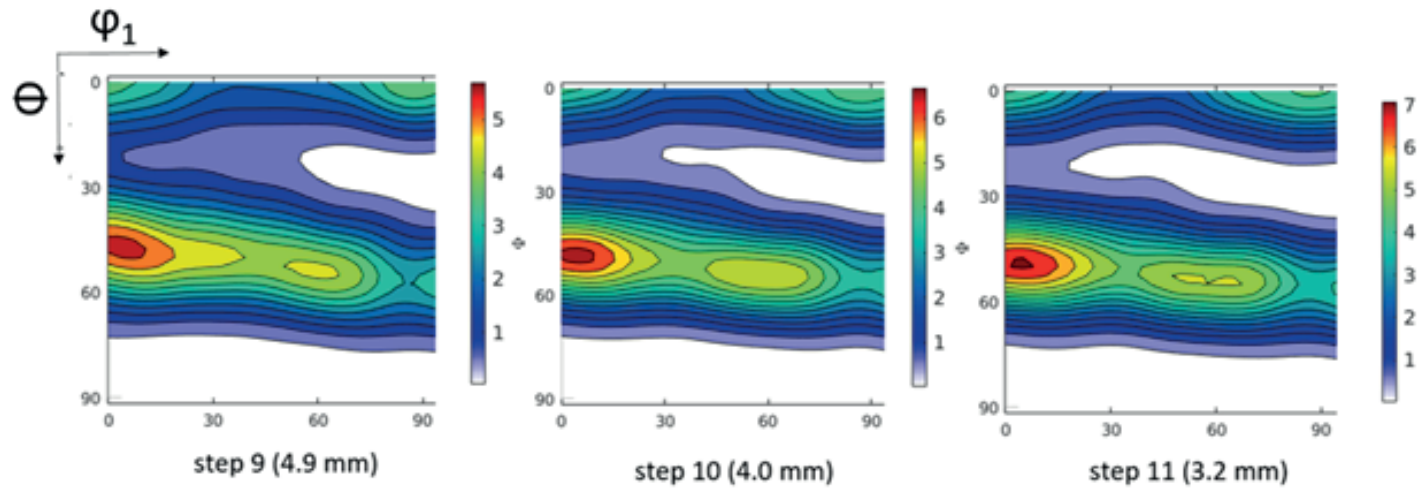

Figure 7: Simulated ODF sections $\varphi_{2}=45^{\circ}$ for rolling steps in ferritic range of temperature (central layer).

In outer layer all rolling steps take place in ferritic temperature range. ODF sections for outer layer are shown on Figure 9. Experimental ODF section for corresponding layer placed on Figure 10.

\section{DISCUSSION}

It can be seen from the graphs in Fig. 5 that the sheet material undergoes an inhomogeneous deformation through the sheet thickness during hot rolling, which is manifested 


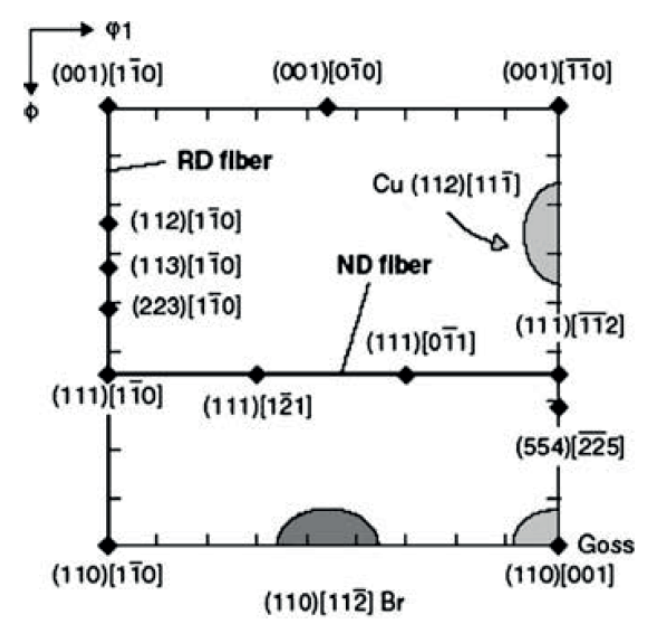

a

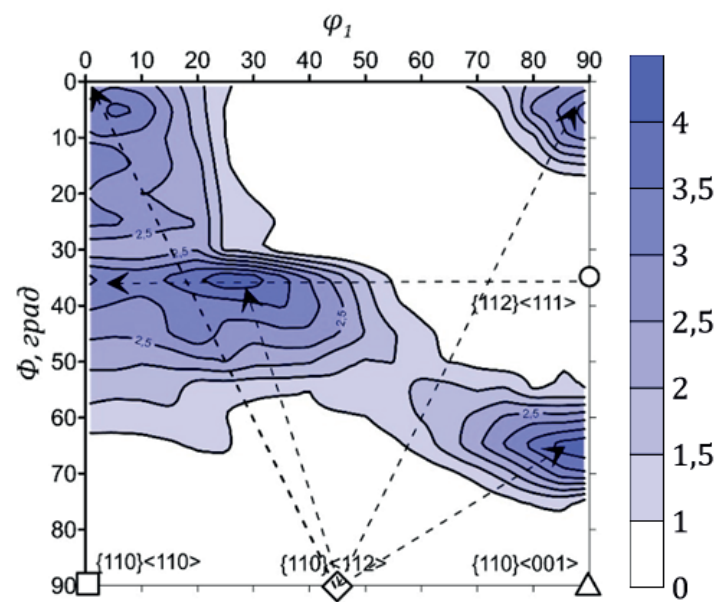

$\mathrm{b}$

Figure 8: Experimental obtained ODF section $\varphi_{2}=45^{\circ}$ for austenite (a) [6] and ferrite (b) in central part of sheet.

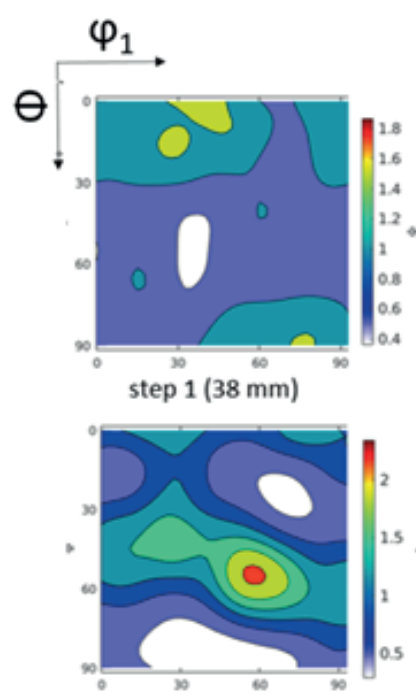

step $5(13.6$

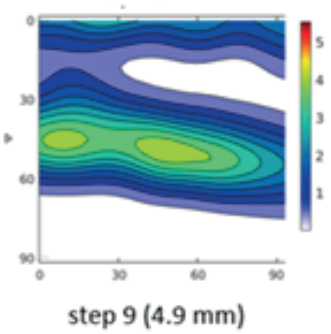

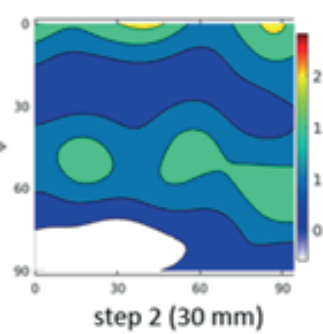

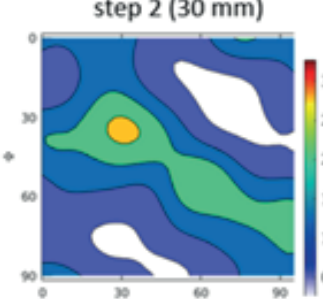

step 6 (10.4 mm)

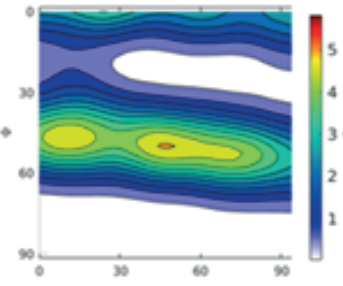

step $10(4.0 \mathrm{~mm})$

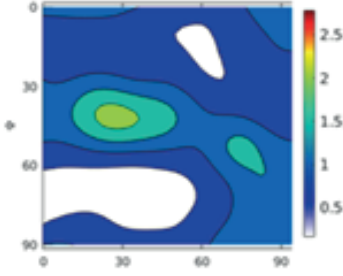

step 3 ( 23

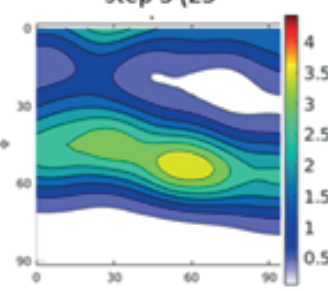

step $7(8.0 \mathrm{~mm})$

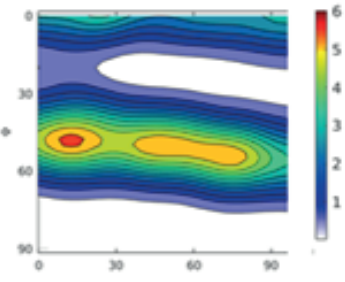

step $11(3.2 \mathrm{~mm})$

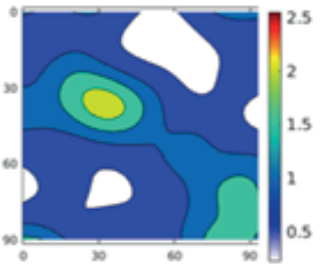

step $4(17.8 \mathrm{~mm})$

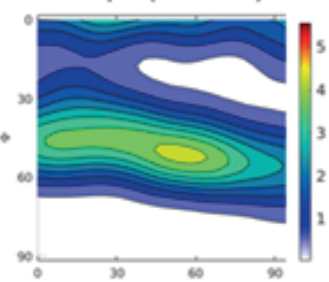

step $8(6.3 \mathrm{~mm})$

Figure 9: Simulated ODF sections $\varphi_{2}=45^{\circ}$ for outer layer.

in the difference between the components of the strain tensor in different layers, i.e. for different values of the parameter $S$. This is due to the fact that the outer layers of the sheet cools down faster than internal layers, and layers with different temperatures have different mechanical properties. Therefore, the difference between the components of the strain tensor is greater at those stages of rolling where there is 


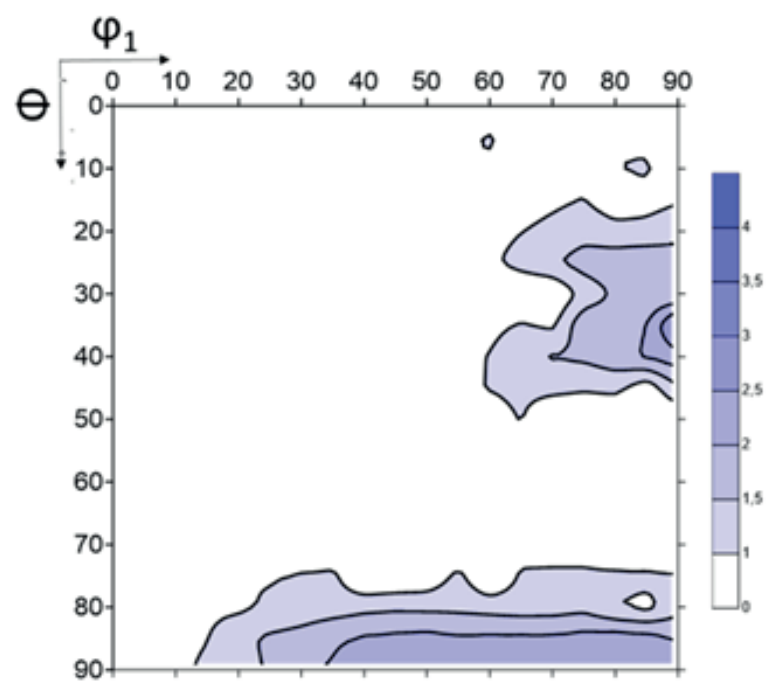

Figure 10: Experimental ODF section $\varphi_{2}=45^{\circ}$ for outer layer.

the greatest temperature difference between the layers of sheet (passes 2-6). In this case, a higher shear component of the strain tensor $\varepsilon_{x y}$ is observed near the surface of the sheet relative to the central part of the sheet. The shear component is larger in magnitude than the normal components $\varepsilon_{x}, \varepsilon_{y}$ (passes 2-4), which is a result of friction in the contact between the roll and the sheet.

The simulated texture components for the austenite $\{110\}<112>,\{110\}<001>$ $\{112\}<111\rangle$ and ferrite $\{100\}<110\rangle,\{111\}<110\rangle,\{554\}<225>$ are in satisfactory agreement with the experimental ones $[1,6]$. A certain difference in the ODF can be explained by the discrepancies in the rolling conditions of the sheet studied experimentally and those used in the simulation.

In the outer layers, the modeled texture components do not correspond to the experimental ones. The resulting texture components are close to the components $\{100\}\langle 110\rangle,\{112\}\langle 110\rangle,\{554\}<225\rangle$, characteristic to the central layer. Thus, a deformation with a shear component $\varepsilon_{x y}$ using the systems $\{110\}<111>$ and $\{112\}$ $<111>$ does not result in the formation of an anomalous texture on the sheet surface.

Possible reasons for the discrepancy between the results of modeling and experiment can be the not considering in the model of the relationship between the texture and the mechanical properties of the material, as well as the likely effect of other deformation mechanisms in addition to crystallographic slip in the systems mentioned above. As other mechanisms, one can consider slip in other systems or grain boundary sliding under conditions of dynamic recrystallization or phase transformations. The proposed model does not consider the possibility of the material surface hardening due to interstitial impurities diffusing from the surface of the sheet during hot rolling, as shown in [3]. 
Thus, in order to obtain a coincidence of the modeling results of the texture formation process in the outer layers of hot-rolled steel sheet with experimental ones, further improvement of the model used and additional study of the deformation mechanisms operating under conditions of hot rolling in outer layer are necessary.

\section{CONCLUSIONS}

1. Modeling of ferritic steel hot rolling at macro- and mesoscopic levels was carried out. It is established that the deformation of the sheet is inhomogeneous in thickness due to the temperature gradient and friction in the contact of the sheet with the rollers.

2. The crystallographic texture components obtained by modeling in the central layers of the hot-rolled sheet satisfactorily correspond with the components of the texture observed experimentally.

3. In the surface layers of a hot-rolled sheet, the modeled components do not agree with the experimental ones, which are anomalous for the deformation of bcc materials.

\section{ACKNOWLEDGMENT}

The reported study was funded by RFBR according to the research project No. 16-3200180 mol_a and was supported by the MEPhI Academic Excellence Project (agreement with the Ministry of Education and Science of the Russian Federation of August 27, 2013, project no. 02.a03.21.0005)

\section{References}

[1] Yu. Perlovich, M. Isaenkova, P. Dobrokhotov, S. Stolbov, O. Bannykh et al AIP Conference Proceedings 1896, 190019 (2017)

[2] O. Engler, C.N. Tomé, M.Y Huh. A study of through-thickness texture gradients in rolled sheets // Metall. and Mat. Trans. A. - 2000. - Vol. 31, No. 9 - pp.2299-2315.

[3] Yu. Perlovich, M. Isaenkova Int J Mater Form (2010) Vol. 3 Suppl 1.

[4] Crystal Plasticity Finite Element Methods. F. Roters, P. Eisenlohr, T.R. Bieler and D. Raabe. - KGaA, Weinheim,2010 - 207 p.

[5] D.D. Tjahjanto et al. 2010 Modelling Simul. Mater. Sci. Eng. 18015006 
[6] Microstructure and Texture in Steels and other materials. A. Haldar, S. Suwas, D. Bhattacharjee (Eds.) - Springer-Verlag London Limited, 2009-488 p. 